\title{
Magnetic properties of soft magnetic composites under three-dimensional
}

\section{excitations}

\author{
Zhi Wei Lin* ${ }^{1}$, Jian Guo Zhu ${ }^{1}$, You Guang Guo ${ }^{1}$, Jin Jiang Zhong ${ }^{1}$, \\ and Shuhong Wang ${ }^{1,2}$
}

${ }^{1}$ Faculty of Engineering, University of Technology, Sydney, PO Box 123, Broadway, NSW 2007, Australia

${ }^{2}$ Faculty of Electrical Engineering, Xi'an Jiaotong University, 28, West Xian Ning Road, Xi'an, 710049, China

\begin{abstract}
Soft magnetic composite (SMC) materials are broadly used in low cost high performance electrical machines due to their magnetic isotropy, low eddy current loss, and capability to mould complex shapes. To optimise the performance of electrical machines, the three-dimensional (3-D) magnetic properties must be well understood and accurately modeled. For this purpose, a 3-D magnetic property measurement system was built and special sensing devices were fabricated. This paper presents, in detail, the magnetic flux density loci and the corresponding magnetic field strength loci for an SMC material when the flux density loci were controlled to be circles lying in the xoy-, yoz-, and zox-plane at $50 \mathrm{~Hz}$.
\end{abstract}

Keywords: 3-D magnetic property, vector hysteresis loci, soft magnetic materials, soft magnetic composites

\section{Introduction}

Soft magnetic composite (SMC) materials are made of ferrous powders. The particles are coated with an insulating organic material to ensure low eddy current loss. A high-pressure compaction process is used to mould the SMC material into a complex shape capable of 
producing three-dimensional (3-D) magnetic flux patterns in electrical machines. In order to improve the machine performance in the design procedure, a series of experimental data and faithful models, which can characterize 3-D magnetic properties of SMC materials, are desired by engineers. In the field of theoretical studies, the experimental data will be helpful to understand the mechanisms of magnetization. To obtain the experimental data, a 3-D magnetic property measurement system was built and special sensing devices were fabricated for a cubic sample. This paper presents, in detail, the magnetic flux density $\mathbf{B}$ loci and the corresponding magnetic field strength $\mathbf{H}$ loci for a cubic sample of SMC material when the $\mathbf{B}$ loci were controlled to be circles lying in the xoy-, yoz-, and zox-plane at $50 \mathrm{~Hz}$, respectively.

\section{Experimental system}

A 3-D magnetic property measurement system was successfully built to magnetize cubic samples of soft magnetic materials [1, 2]. A special H and B sensing house, which consists of six $\mathrm{H}$ coils with embedded planar B coils for sensing the tangential component of $\mathbf{H}$ and normal component of $\mathbf{B}$ at the six sample surfaces, was fabricated and calibrated to measure the $\mathbf{H}$ and $\mathbf{B}$ loci. Fig. 1 shows the schematic diagram of the system and a photo of the sensing house. The sample material in this study is an SMC material made of highly pure iron powder with surface coating provided by Höganäs AB, Sweden. The dimensions of the sample are 22 $\times 22 \times 22 \mathrm{~mm}^{3}$. Six thin $\mathrm{H}$ coils with embedded B coils were attached to the cubic sample to measure the sample $\mathbf{H}$ and $\mathbf{B}$ vectors. For easy installation six $\mathrm{H}$ coils were integrated into a rigid sensing house in which the sample was placed. All coils were carefully calibrated in a long solenoid to obtain the coil coefficients, which were used to calculate the magnetic field at the sample surface. The details are presented in reference [3]. 


\section{Results and discussion}

Fig. 2 shows the hysteresis loops while the sample was magnetized at $50 \mathrm{~Hz}$ in $3-\mathrm{D}$ magnetization system along the $x$-, $y$-, and $z$-axis, respectively. Fig. 3 shows the magnetic power loss along these three axes. The results are consistent with the data obtained previously by 2-D magnetic tester and those provided by the manufacturer. It is noticed that properties measured along the $x$ - and $z$-axis are similar while the $y$-axis seems to be an easy direction for magnetization. This slight anisotropy might be caused by the compaction process though the sample is supposed to be isotropic. The SMC materials are composed of iron particles with irregular shapes, and the total power loss of the sample along one direction is a summation of losses dissipated by each particle, which are dominated by particle magnetocrystalline structure, magnetostriction, stress, shape, impurities and defects. These factors are expected not to be impacted significantly during the hot compression as the collective coercivities along three directions are the same. As a result, the losses along three directions are similar in despite of a present of slight difference caused by slight misalignment of yokes of 3-D measurement system. However, the compression causes non-uniform distribution of mass density. Morphologies observed using optical microscope display that the interstice rate at the compressing surface is $22 \%$ while it is $11 \%$ at the side surface. It means that the particles are much closer along the $y$-direction than along the $x$ - and $z$-directions, that is, high mass density along the $y$-direction. As a result, a smaller field can reach higher flux density along $y$ direction comparing with $x$ - and $z$ - directions. It is the reason that the $y$-direction is an easy direction for magnetization, along which the permeability is relatively higher.

Fig. 4 shows the $\mathbf{H}$ and $\mathbf{B}$ loci while the $\mathbf{B}$ loci were controlled to be circles with different amplitudes up to $1.3 \mathrm{~T}$, in the xoy-, yoz- and zox-plane at $50 \mathrm{~Hz}$, respectively. It is shown that 
the $\mathbf{B}$ loci are well controlled while the $\mathbf{H}$ loci have some aberrance, that is, they evolve from circle at lower flux densities into rectangle at higher densities. Fig. 5 shows the magnetic power loss values in three different planes. The rotational magnetic power loss is larger than the alternating loss.

\section{Conclusion}

The 3-D magnetic properties of a soft magnetic composite material have been measured using a novel 3-D magnetic property measurement system at $50 \mathrm{~Hz}$. For alternating magnetic properties the results are consistent with our previous data and those provided by the manufacturer. For 3-D properties, the loci of magnetic flux density and field strength, and magnetic power losses have been measured and discussed when the flux density loci were controlled to be circles lying in three orthogonal planes.

\section{References}

[1] J. G. Zhu, J. J. Zhong, Z. W. Lin, and J. D. Sievert, Measurement of core losses with 3D magnetic fluxes, IEEE Transactions on Magnetics 39(2003), 3429-3431.

[2] Z. W. Lin, H. W. Lu, J. G. Zhu, J. J. Zhong, X. L. Wang, and S. Y. Ding, Vector characterization of soft magnetic materials, Journal of Applied Physics 97(2005), 10R306-13.

[3] Z. W. Lin, J. G. Zhu, Y. G. Guo, J. J. Zhong, and H. W. Lu, B and H sensors for 3-D magnetic property testing, presented at International Symposium on Interdisciplinary, 
Electromagnetic, Mechanic \& Biomedical problems ISEM 2005, September 12-14, 2005, Bad Gastein (Salzburg), Austria, 2005. 


\section{Figure captions}

Fig. 1. Diagram of the 3-D measurement system (left side) and a photo of the sensing house (right side).

Fig. 2. Hysteresis loops while the sample was magnetized along $x$-direction (left), $y$-direction (middle), and z-direction (right).

Fig. 3. Magnetic loss values for alternating magnetizations on the $x-, y$-, and $z$-axis.

Fig. 4. Round B loci (left column) and corresponding $\mathbf{H}$ loci (right column) in the xoy-plane (top row), yoz-plane (middle row), and zox-plane (bottom row). (a): round B loci in the xoyplane. $\mathrm{B}=0.22,0.43,0.65,0.83,1.04,1.20$, and $1.32 \mathrm{~T}$. (b): round $\mathbf{B}$ loci in the yoz-plane. $\mathrm{B}$ $=0.20,0.39,0.59,0.79,0.96,1.13$, and $1.29 \mathrm{~T}$. (c): round $\mathbf{B}$ loci in the zox-plane. $\mathrm{B}=0.21$, 0.42, 0.61, 0.81, 1.00, 1.16, and $1.29 \mathrm{~T}$.

Fig. 5. Magnetic loss values for round $\mathbf{B}$ loci in the xoy-, yoz-, and zox-plane. 
Figure 1 (side by side)
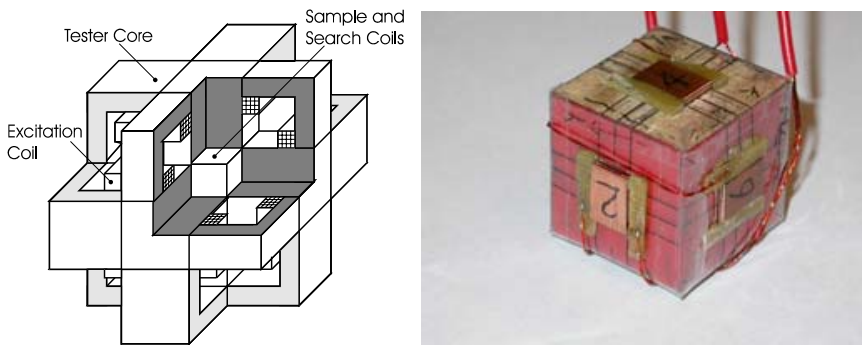
Figure 2

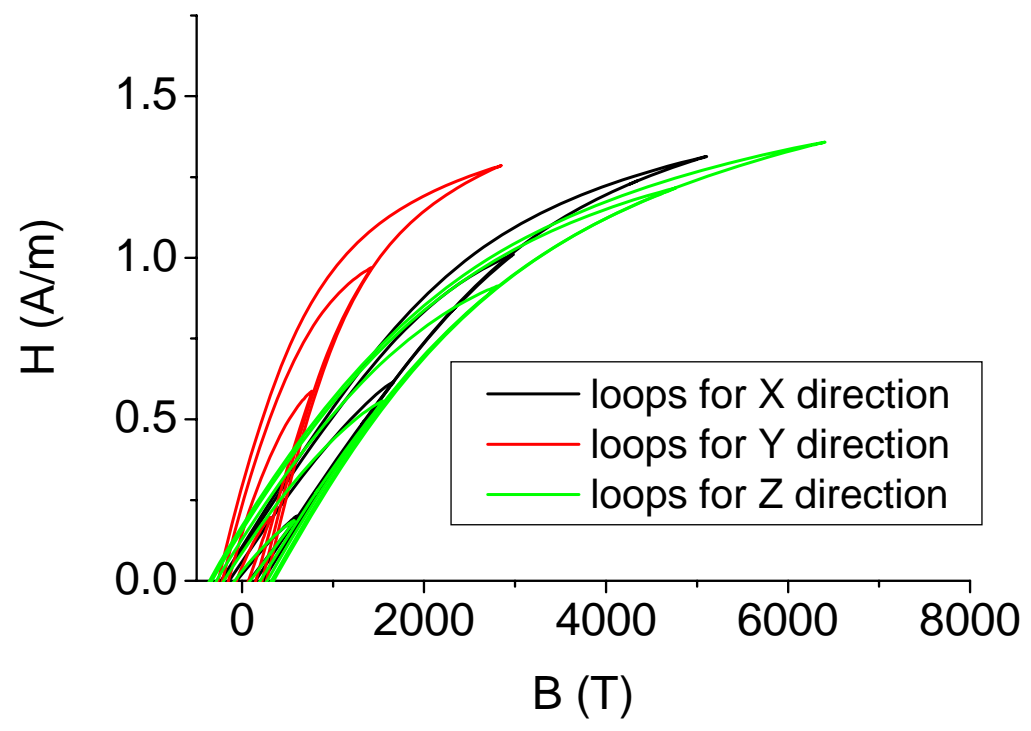


Figure 3

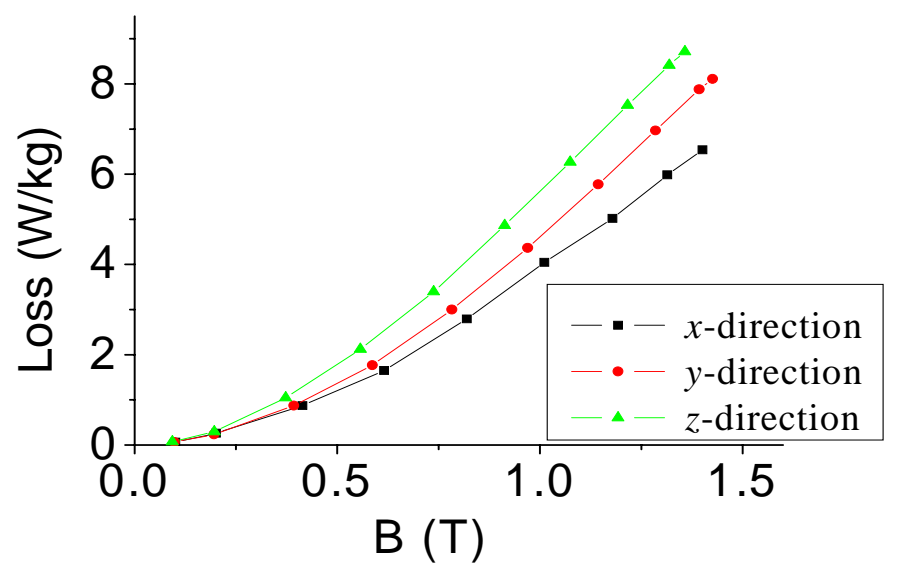


Figure 4

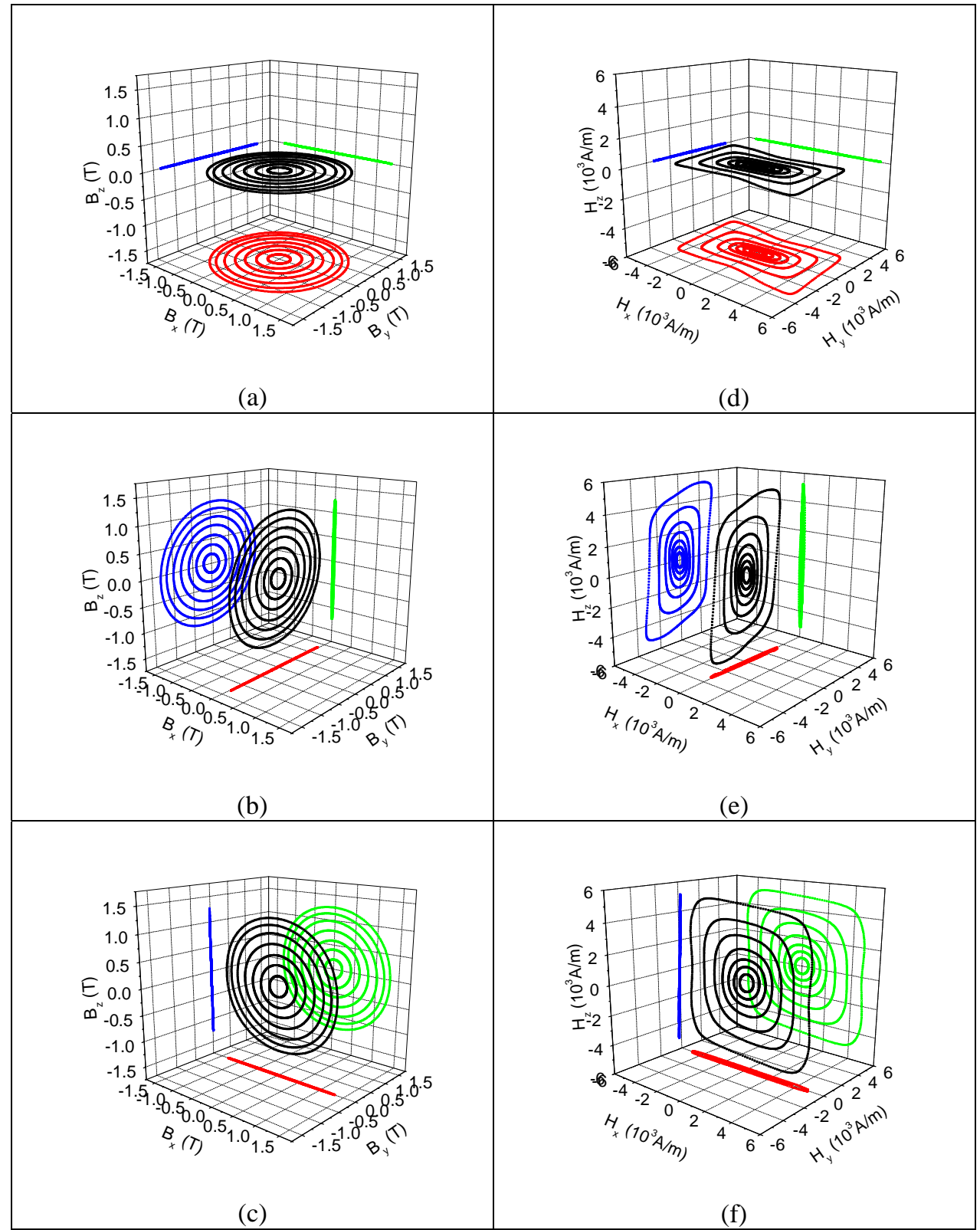


Figure 5

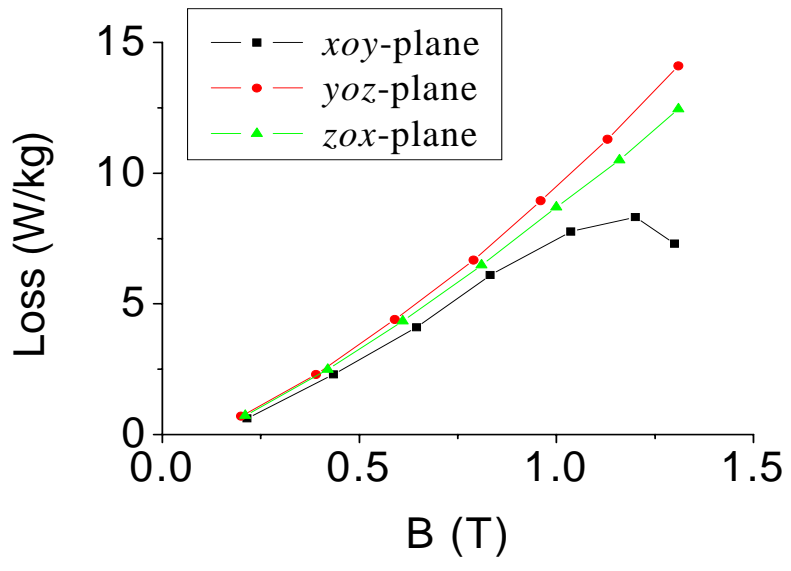

